\title{
Analysis of power quality in presence of frequency distortions
}

\author{
Violetta Zatsepina ${ }^{1}$, Evgeniy Zatsepin ${ }^{1}$, Oleg Shachnev ${ }^{1}$, Alexander Shachnev ${ }^{2}$, and Timur Petrov , $^{*}$ \\ 1Department of electrical equipment, Lipetsk state technical University, Lipetsk, 30 Moskovskaya street, 398055, Russia \\ ${ }^{2}$ Department of housing and communal services of Lipetsk city administration, Lipetsk, 8 Kommunalnaya square, 398000, Russia \\ ${ }^{3}$ Kazan State Power Engineering University, Kazan, Russia
}

\begin{abstract}
Today, semiconductor elements are becoming more popular in power supply networks. Their presence in any objects of electrical equipment provides high performance with high operating efficiency. However, modern research shows the semiconductor element base negatively affects quality of electricity when they are used in parallel. In low- and medium-voltage networks, this is fraught with the appearance of frequency «noise», which negatively affects parameters equipment with presence of latter, as well their control system. To detect them, a special approach is necessary, since such harmonics can appear both from directly semiconductor elements and from operating mode of equipment where element base is applied. The purpose of this article identifies higher harmonic distortions generated by objects containing semiconductor elements.
\end{abstract}

\section{Theoretical substantiation}

Modern electric power industry is closely connected with the use of semiconductor element base. It is used in various converters, automation, sensors and other elements where performance, reliability and high technical and economic indicators are required. It is known that the efficiency of their operation depends on the quality of electricity, since the vast majority of semiconductors must comply with the voltage level, and even a deviation from the normal voltage level within the permissible $5 \%$ can reduce their efficiency and reliability, which is not rational for conveyor production and utilities [1].

Reducing the probability of failure semiconductor elements lies in comprehensive analysis of operating voltage level and identification of higher harmonic components with their subsequent elimination. The study is useful in low- and medium-voltage objects that are widely used and where their compensation unlikely. It should also be taken into account that in control rooms for monitoring voltage level in cells, a graph of active values is often used, which does not give staff an understanding level of harmonics, since averaging also affects «white noise», so for greater objectivity, the article will use graphs instantaneous values of electrical quantities.

\section{Practical experiment and explanations}

We will conduct an experiment in Matlab R2014a for an electric network with voltage of $0.4 \mathrm{kV}$ with a diodetype lighting system, where the primary voltage converters (drivers) often fail. The arguments of standard Matlab function of signal itself, dimension of result vector (FftL), and measurement. The last argument defines which dimension signal is located along if input a multidimensional array. Since signal under study is vector, it can be omitted. Consider first the electrical load without consumers. As result, we get a vector of complex numbers. This is exponential representation of our signal in frequency domain $[2,3]$.

The modules of these complex numbers represent amplitudes of corresponding frequencies, and arguments represent their initial phases. If resulting phase is uniquely calculated in radians or degrees, then additional tools must be used with amplitude and frequencies, which we will discuss later [4, 5].

For a clear comparison, let's give a graph with disconnected consumers (fig. 1, upper graph) and connected ones (fig. 1, lower graph). Below, we present the decomposition according to fast Fourier transform in amplitude-frequency spectrum in fig. 2 (lower and upper graph, respectively). Note that this decomposition is useful in case of continuous analysis of current charts and their dynamic change will be presented as quickly as possible, since it affects the minimum amount of resources.

The current analysis used a sample rate of $F_{d}=1024$ $\mathrm{Hz}$, and the number of Fourier lines in construction of spectrum - 5120. To ensure higher accuracy, the $F_{d}$ indicator can be taken higher, but this is fraught with obtaining imaginary frequency perturbations, since calculation in Matlab R2014a is performed in a discrete region [6].

After receiving results in figures 1 and 2 , it is not difficult to notice that frequency disturbances are visible in spectral region, but signal from them is quite weak, and in case of analyzing graphs active values, it is almost not noticeable due to data averaging.

* Corresponding author: tobac15@ mail.ru 


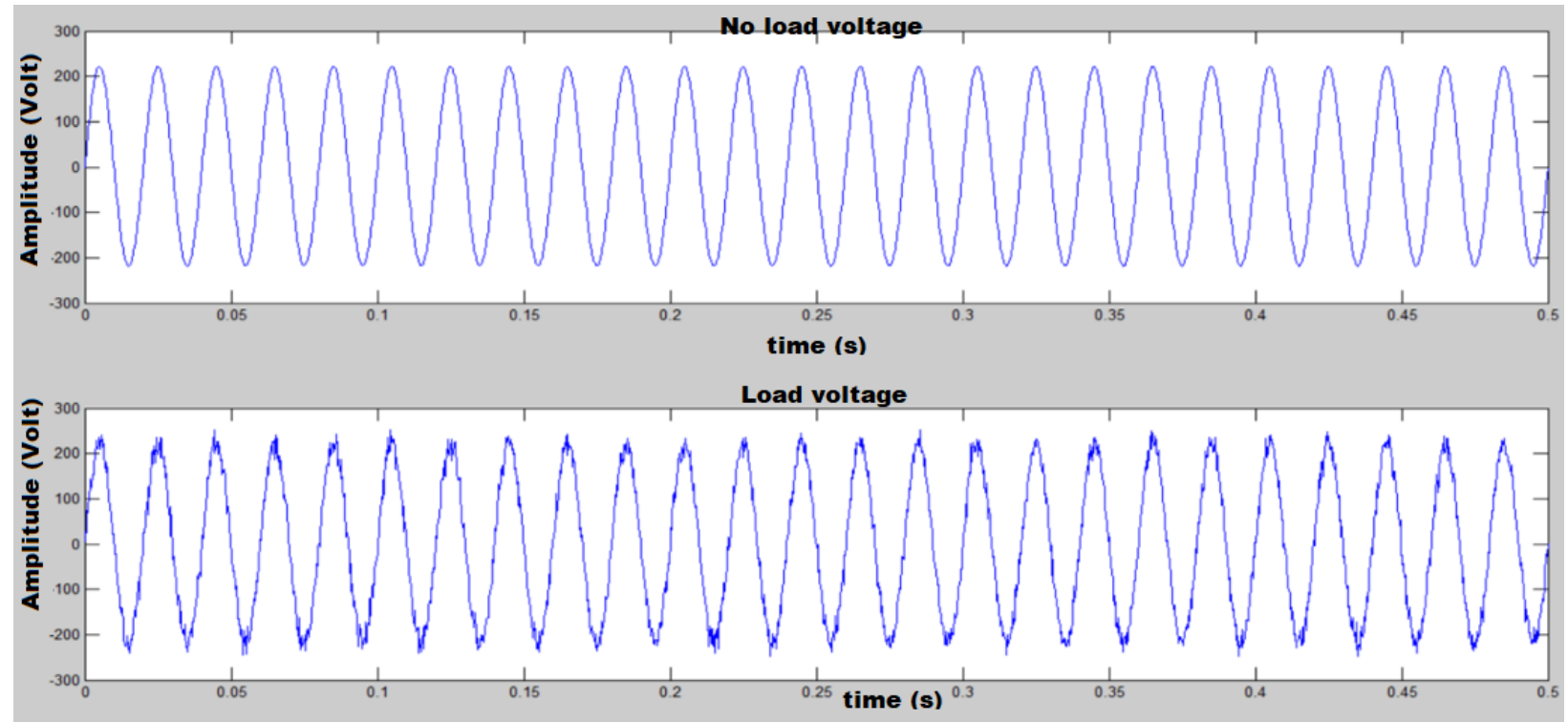

Fig. 1. Voltage graphs without load (upper) and with load (lower).

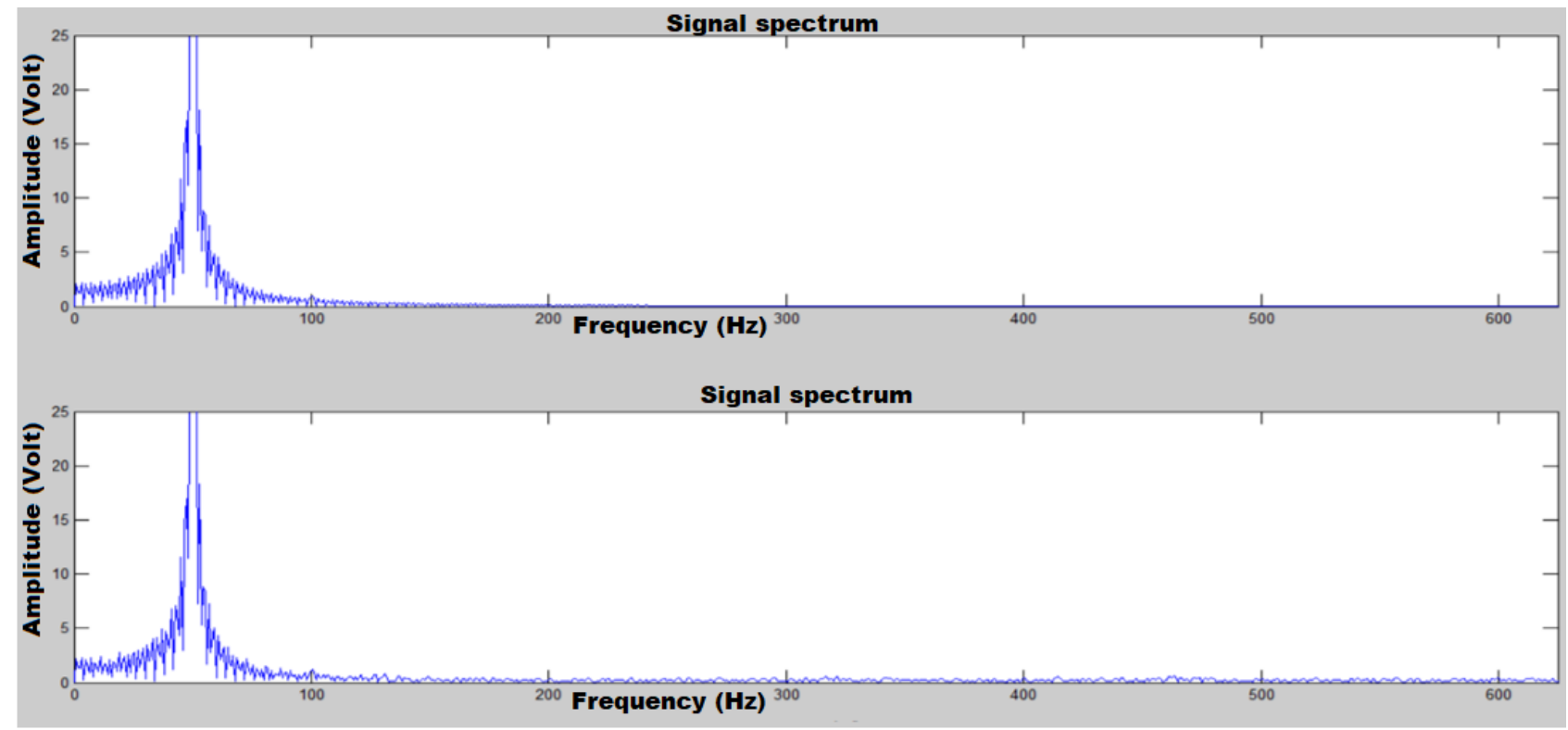

Fig. 2. The amplitude-frequency spectrum without load (top) and with load (bottom).

So, if there is a main graph with a frequency of 50 $\mathrm{Hz}$, you can see fluctuations in 3, 5, 7, 9 (especially clearly), 11 (faintly noticeable) order, which corresponds to frequencies in $150 \mathrm{~Hz}, 250 \mathrm{~Hz}, 350 \mathrm{~Hz}, 450 \mathrm{~Hz}, 550$ $\mathrm{Hz}$. It is possible to determine the frequency level from spectral signal, but it is quite difficult to determine amplitude of each order. To get out of this situation, it is advisable to use either other Fourier transforms, which is fraught with large resources and will affect calculation time, or use scaling coefficients, which are used by default in wavelet decomposition $[7,8]$.

We will conduct an experiment using a wavelet transform of the current signal. Note that 2025 points obtained over time $\mathrm{t}=[0 \ldots 0.5]$ were taken for analysis. Since analysis uses a one-dimensional graph, we will use section Wavelet 1-D in paragraph of calling wavemenu Matlab R2014a (Wavelet Toolbox). Given that it is planned to use real-time wavelet analysis, the expansion to third level by Daubechies wavelets (db10) will be sufficient. Figure 3 shows the complete decomposition of the studied graph of the lighting network voltage [9].

Since graph is one-dimensional, the study does not have a time domain and takes the numbering of points. You can link to time by writing additional scripts, but this is not included in this study. Figure 3 shows that current graph $\mathrm{S}$ is decomposed into final approximated graph $a_{3}$ and detailed graphs $d_{1}, d_{2}, d_{3}$. In discrete domain, addition of all graphs will give the initial one, therefore, the probability of extra frequencies appearing during conversion is very small, which is very important when analyzing this kind of load graphs [10].

In order to determine frequency indicators, it is necessary to take inversely proportional values of scale the distribution histogram according to figure 4 on original graphs.

Here $\mathrm{S}$ is histogram of initial graph under study, $\mathrm{a}_{3}-$ is the histogram of final approximated graph with an enlarged scale, as evidenced by exit of abscises axis 


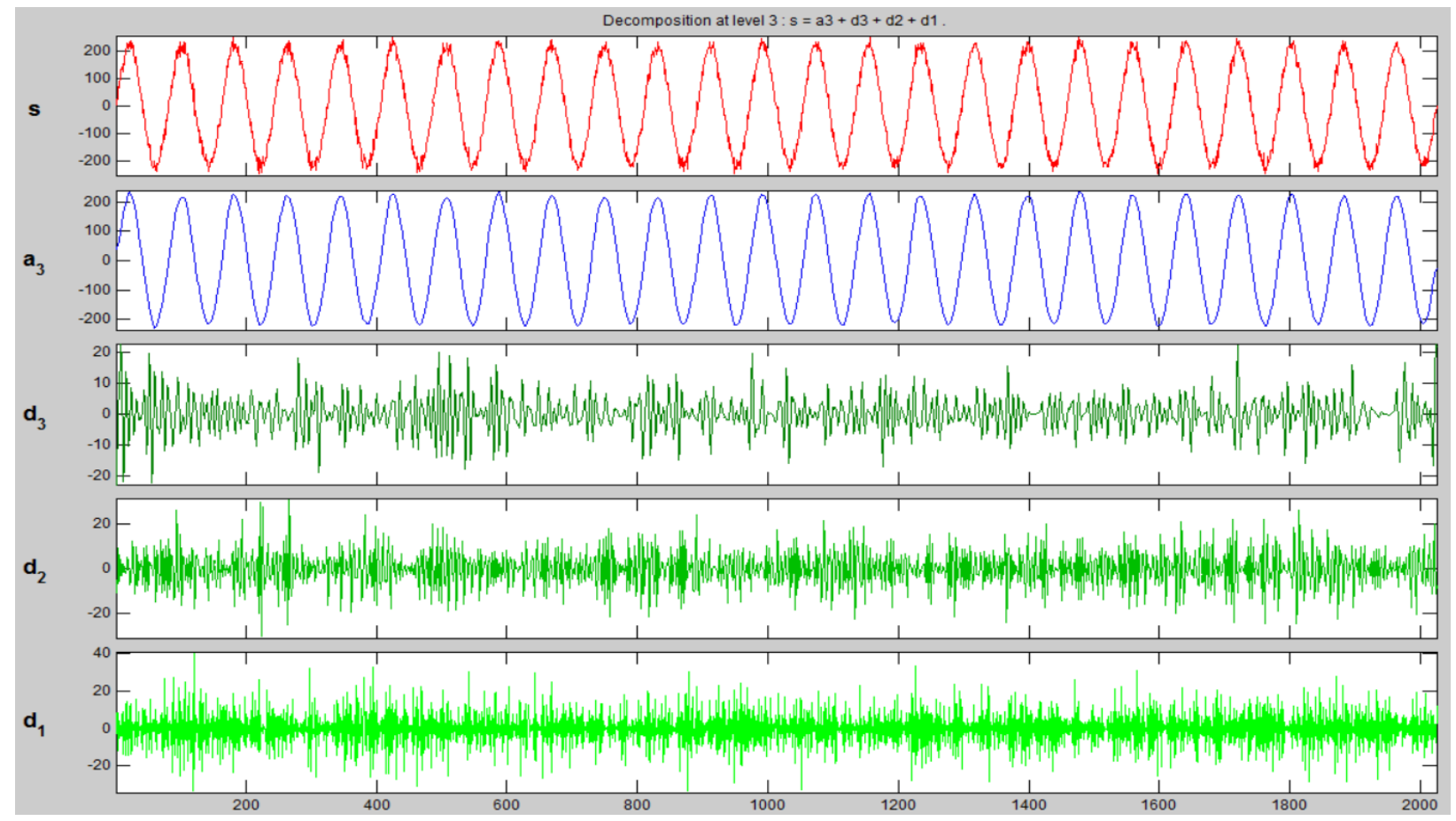

Fig. 3. The decomposition graph of voltage on Daubechies wavelets to third level.
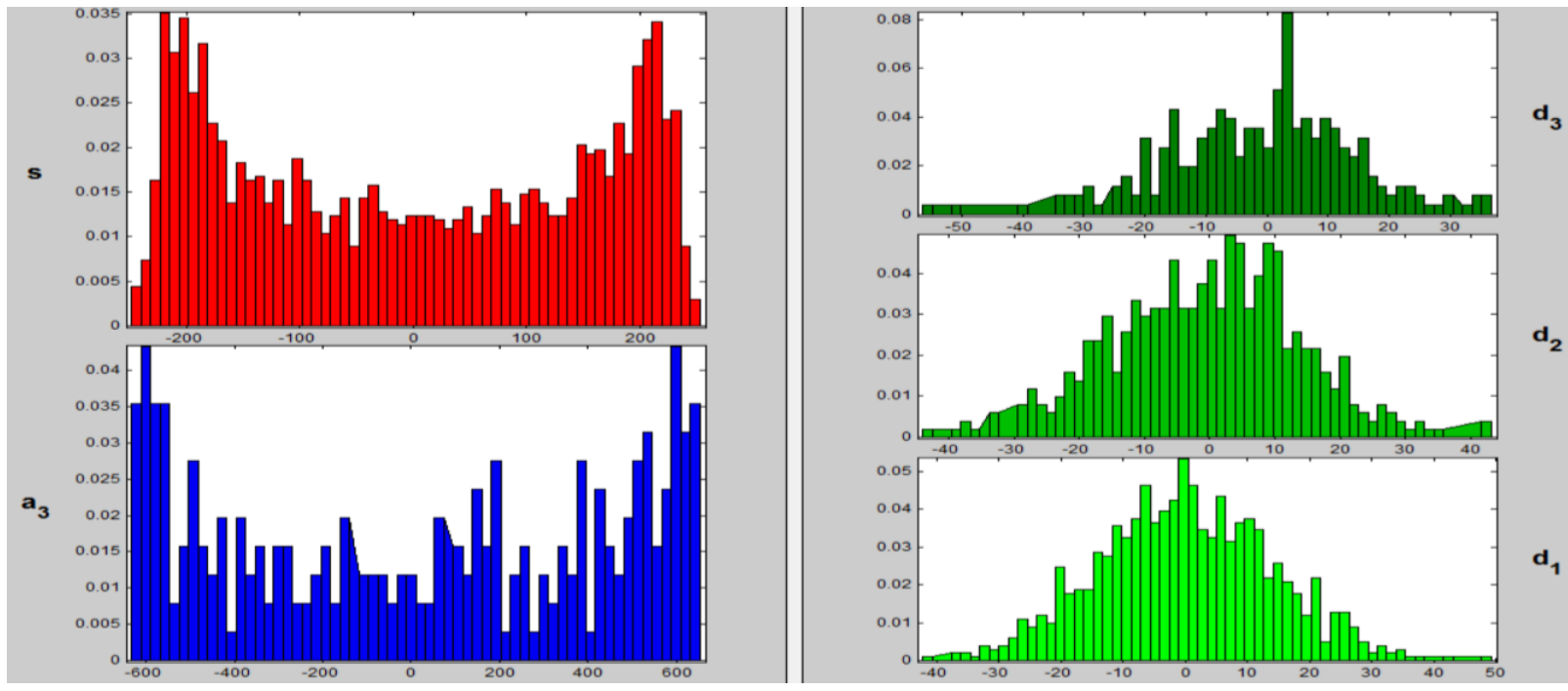

Fig. 4. Histograms of wavelet expansion graph under study.

beyond boundaries of values of 220 amplitude components; $\mathrm{d}_{1}, \mathrm{~d}_{2}, \mathrm{~d}_{3}-$ corresponding level of decomposition histogram of detailing graphs, as evidenced by low scale values. For example, most of high-frequency bursts were identified at first decomposition corresponding $\mathrm{d}_{1}$ graph, which corresponds to scale $0.0015 \ldots 0.01$ according to histogram of amplitude 20-40. Taking inversely proportional values, we get frequency characteristics, that is, the corresponding $666.67 \ldots 100 \mathrm{~Hz}$. You can get more accurate frequency values by calculating each column of histogram [11-14].

\section{Processing of results and conclusion}

Comparing the results obtained with fast Fourier transform and wavelet analysis, we can note the following:

- wavelet analysis provides more accurate indicators for frequency perturbations;

- wavelet analysis is more in demand when loads change or when transient modes occur;

- data of frequency characteristics in wavelet analysis can be used in automated control systems due to high performance;

- wavelet analysis is difficult to understand, so it is possible to use wavelets Daubechies or Haar (the most simple when translated into the usual frequency characteristics); 
- Fourier analysis is useful for surface analysis of load graphs in the absence of weak frequency perturbations.

Semiconductor elements that generate mostly «white noise» are poorly suited for the usual Fourier analysis. Its low accuracy may affect the failure of semiconductor voltage sensors that are used in systems of APM, smart home, smart city, lean manufacturing, and others [1518]. Given that above things are being developed and placed everywhere, elimination of frequency noise and its diagnosis will be a priority in near future. Using method described in article will allow you to diagnose and further take measures to eliminate frequency disturbances, which will have a positive impact on their technical and economic indicators [19, 20].

\section{References}

[1] V.I. Zatsepina, E.P. Zatsepin, O.Ya. Shachnev, Improving Eefficiency of High-Power Plants through Modernization STATCOM Devices, Proceedings - 2019 1st Int. Conf. on Control Systems, Mathematical Modelling, Automation and Energy Efficiency, SUMMA 2019, 1-5 (2019)

[2] Z. Dianmin, D. Shijie, G. Xiaohong, ECommercial-e is applied on market modem Power Syst. Machine, 11-14 (2001)

[3] V.I. Zatsepina, E.P. Zatsepin, O.Ya. Shachnev, Analysis of load indicators power grid complex when feeding differentiated consumer, Int. Conf. on Industrial Engineering Application and Manufacturing (ICIEAM), 8742998 (2019)

[4] L. Fernández-Robles, G. Azzopardi, E. Alegre, N. Petkov, Machine-vision-based identification of broken inserts in edge profile milling heads, Robotics and Computer-Integrated Manufacturing, 276-283 (2017)

[5] A.N. Shpiganovich, A.A. Shpiganovich, O.V. Fedorov, Concerning the opportunity of fail-safe systems development, Int. Conf. on Industrial Engineering Application and Manufacturing (ICIEAM), 8076255 (2017)

[6] E.I. Gracheva, O.V. Fedorov, Forecasting reliability electrotechnical complexes of in-plant electric power supply taking into account lowvoltage electrical apparatuses, Int. Conf. on Industrial Engineering Application and Manufacturing (ICIEAM), 8743057 (2019)

[7] T.I. Petrov, A.R. Safin, I.V. Ivshin, A.N. Tsvetkov and V.Y. Kornilov, The Prospects of Using a Synchronous Machine with Permanent Magnets in the Oil Industry, 14th International Scientific-Technical Conference on Actual Problems of Electronic Instrument Engineering, APEIE 2018 - Proceedings, article no. 8546157, 336-338 (2018)

[8] E. Gracheva, A. Alimova, Calculation methods and comparative analysis of losses of active and electric energy in low voltage devices, Proceedings - 2019 Int. Ural Conf. on Electrical
Power Engineering, UralCon 2019, 361-367 (2019)

[9] V.I. Zatsepina, E.P. Zatsepin, Reliability of power supply systems under influence of negative factors, Int. Conf. on Industrial Engineering Application and Manufacturing (ICIEAM), 1-4 (2017)

[10] E.I. Gracheva, A.N. Alimova, Relationship between the characteristics of power transformers and their load capacity, News of higher educational institutions in Chernozem region $\mathbf{1}$, 48-55 (2019)

[11] O.V. Fedorov, Some features of structure internal systems power supply, Reliability and security of energy 3, 30-33 (2015)

[12] B.N. Parsunkin, O.V. Petrova, E.I. Polukhina, Research influence of electric mode DSP on cost produced steel, Theory and technology of metallurgical production, 44-46 (2014)

[13] A. Kiyoumarsi, M. Ataei, R. Hooshmand, Electric Arc Furnace Voltage Flicker Mitigation by Applying a Predictive Method with Closed Loop Control of TCR/FC Compensator, Journal of Electrical Engineering \& Technology 1, 116-128 (2010)

[14] E.I. Gracheva, O.V. Naumov, Evaluation criteria of contact group technical state concerning electrical appliances, International Journal of pharmacy and Technology 4, 27084-27091 (2016)

[15] D. Maihöfner, I. Talavera, J. Hanson, C. Bott, Vertical reactive power flexibility through distributed energy resources for a reactive energy management, 11th IEEE Int. Conf. on Compatibility Power Electronics and Power Engineering, 100-105 (2017)

[16] L.S. Czarnecki, P.D. Bhattarai, A Method of Calculating LC Parameters of Balancing Compensators for AC Arc Furnaces, IEEE Transactions on Power Delivery 32, 688-695 (2017)

[17] W. Bialy, V.E. Sovin, V.I. Zatsepina, E.P. Zatsepin, O.Ya. Shachnev, Ensuring efficient operation of electromechanical systems with frequency regulation with periodic voltage sags, E3S Web of Conferences, 05059 (2019)

[18] V.V. Moroshkin, V.A. Skopintsev, Analysis and forecast of emergency situations in electric power systems based on statistical methods, Electric power plants, 12, 2-6 (2012)

[19] M.T. Esfahani, B. Vahidi, A Predictive Reactive Power Measuring Based on Time Series and DLSL Algorithm for Compensating Applications, IEEE Transactions on Instrumentation and Measurement 64, 2646-2656 (2015)

[20] J. Wang, A wavelet denoising method based on the improved threshold function, Int. Conf. on wavelet Analysis and Pattern Recognition, 70-74 (2014) 